\title{
Model Based Control of Moisture Sorption in a Historical Interior
}

\author{
P. Zítek, T. Vyhlídal
}

\begin{abstract}
This paper deals with a novel scheme for microclimate control in historical exhibition rooms, inhibiting moisture sorption phenomena that are inadmissible from the preventive conservation point of view. The impact of air humidity is the most significant harmful exposure for a great deal of the cultural heritage deposited in remote historical buildings. Leaving the interior temperature to run almost its spontaneous yearly cycle, the proposed non-linear model-based control protects exhibits from harmful variations in moisture content by compensating the temperature drifts with an adequate adjustment of the air humidity. Already implemented in a medieval interior since 1999, the proposed microclimate control has proved capable of permanently maintaining constant a desirable moisture content in organic or porous materials in the interior of a building.
\end{abstract}

Keywords: equilibrium moisture sorption, humidity control, non-linear cascade control.

\section{Introduction}

Most materials of sculpture works, paintings, plasters, stuccos, etc., have absorbed a moisture content that settles at an equilibrium level corresponding to the relative humidity and temperature of the surrounding air (Cassar, 1995). Wood is one of the most sensitive materials, furthermore with considerably different mechanical properties and extensibility in each of its three primary axes. Variations in moisture content therefore result in stresses leading to deformations or even to cracks opening in the exhibits (Camuffo, 1998). The internal environment standard in leading galleries and museums involves keeping constant both the interior temperature and the air humidity in the exhibition rooms, but due to impracticable costs, such air-conditioning cannot realistically be implemented as far as remote historical objects are concerned (Cassar, 1993). Owing to this, an invaluable part of European cultural heritage is more or less exposed to the damaging influence of varying moisture caused by air humidity fluctuations (Kotterer, 2002). However, when it is not possible to keep constant both air temperature and humidity, the primary goal is to avoid dimensional extensions or shrinking of the exhibits arising from variations in air humidity and temperature. In principle, it is possible to accept modest temperature changes if they are compensated simultaneously by some adequate air humidity adjustments. In this way it is possible to protect the exhibits against the impact of moisture change and to fulfil the crucial conservation requirement, particularly with respect to the usually anisotropic character of these phenomena. Apparently, this kind of compensation can meet the preservation demands only if both the temperature and the air humidity changes are smooth and slow enough for the internal atmosphere to remain in an almost steady state and the absorbed moisture in the exhibit materials to remain constant.

\section{Air humidity and equilibrium moisture content}

In view of the preservation demands it is a hard task to define unambiguously the proper parameters of the desirable environment. First of all, it is not the visitor s comfort, but rather the benefit of the exhibits that should be the priority in exhibition rooms (Camuffo et al., 2002). Recent research in preventive conservation has introduced the complex concept of the microclimate of a room, in which monuments or exhibits are placed (Camuffo, 1998). Among the numerous parameters of the microclimate, air humidity and temperature are considered as primary and as the most important attributes of the microclimate (Cassar, 1993). Particularly in remote sites of deposits, where neither heating nor air handling devices are in operation, the humidity impact is the most dangerous exposure from the preservation point of view. The decisive role of moisture sorption impact is typical for most of the materials that artistic works are made of, i.e. wood, paper, parchment, leather, ivory, bone, paints, plaster, stucco or stones containing abundant clay minerals, etc. These materials absorb a specific amount of water and are rather sensitive to variations in it. The steady value of this amount, corresponding to the surrounding air humidity and temperature, is called the equilibrium moisture content (EMC), usually expressed as the ratio of the mass of water to the mass of anhydrous material. After the ambient temperature or humidity have changed their values, the absorbed moisture content changes accordingly (Massari, 1993). An increase in EMC increase is then followed by swelling of the material and, conversely a decrease results in contraction. Due to the non-isotropic character of these size changes, harmful deformations or destructive cracks appear as a result. The material extensions resulting from growing EMC are relatively high, and it is important to note that this extension is much higher than just the thermal extension of the dry wood.

In fact the expansion phenomenon is somewhat more complex. For example, a rise in temperature induces thermal extension of dry wood alone, but consequently it results in a drop in relative humidity and therefore also in a drop in EMC, which brings about a material contraction, and vice-versa (Camuffo, 1998). In this way, thermal expansion and EMC contraction are of opposite character and the shrinkage is partially mitigated by the expansion. However, the dimension change due to relative humidity is largely dominant, since only the temperature expansion itself is more than ten times weaker than that of relative humidity (Kowalski, 2003). 


\section{Equilibrium moisture content models}

For each of the considered materials the equilibrium moisture content settles at a level appropriate to the ambient air humidity and temperature. Although the EMC levels are different for various materials, the following properties are common to all of them

- the EMC always increases with growing $\varphi$ and decreases with growing $T$,

- the EMC value is much more sensitive to changes in air humidity than to variations in temperature.

The relationship between EMC as $u$, and both air temperature $T$ and relative humidity $\varphi, u=\Psi(\varphi, T)$ has been fitted by several formulae developed for various areas of application. Usually this relationship is plotted in the coordinates $\varphi$ and $u$, as so-called sorption isotherms, with temperature considered as a parameter. In particular the mathematical models by Day and Nelson and by Simpson (Ball et al., 2001) were found to fit well the experimental data for professionally evaluating EMC. The Day and Nelson model modified by Ball et al. (2001) is of the form

$$
\begin{aligned}
& u=\left[\frac{-\log (1-\varphi)}{A_{D N}\left(T_{K}\right)}\right]^{1 / B_{D N}\left(T_{K}\right)}, \\
& A_{D N}\left(T_{K}\right)=\exp \left(k_{1}\right)\left(\frac{T_{K}}{300}\right)^{v}, \\
& B_{D N}\left(T_{K}\right)=\exp \left(k_{2}\right)\left(\frac{T_{K}}{300}\right)^{\mu},
\end{aligned}
$$

where $T_{K}$ is the temperature in degrees Kelvin, $\varphi$ is the relative humidity expressed as the mass ratio and $k_{1}, k_{2}, \mu, v$ are model parameters. The Simpson model, in the version for wood moisture evaluation is as follows

$$
u=\frac{1800}{W}\left[\frac{K_{0} \varphi}{1-K_{0} \varphi}+\frac{K_{0} K_{1} \varphi+2 K_{0}^{2} K_{1} K_{2} \varphi^{2}}{1+K_{0} K_{1} \varphi+K_{0}^{2} K_{1} K_{2} \varphi^{2}}\right],
$$

where the parameters $W, K_{0}, K_{1}, K_{2}$ are given as quadratic polynomial functions of temperature $T$. Using models (1) or (2), high accuracy can be achieved in fitting the experimental data. However, for the microclimate control idea proposed below these models are less suitable because their derivatives result in fairly complicated formulae. That is why we chose the following Henderson model with only three parameters

$$
u=\left[\frac{-\ln (1-\varphi)}{A(T+B)}\right]^{C}=\Psi(\varphi, T),
$$

to derive the following model based controller of moisture sorption. In this model the relative humidity is considered as the mixing ratio $\varphi \in\langle 0,1\rangle$, temperature $T$ and parameter $B$ are in ${ }^{\circ} \mathrm{C}, C$ is a positive dimensionless exponent, $A$ is a parameter in ${ }^{\circ} \mathrm{C}^{-1}$ and $u$ is the dimensionless ratio of moisture mass content to the mass of dry material.

As regards the function $\Psi($.$) the mutual dependence$ between the variables $\varphi$ and $T$ should be noted. If $x$ is the absolute humidity in $\mathrm{kg}$ of water per one $\mathrm{kg}$ of dry air, its value, corresponding to a certain given $\varphi$ and $T$, results, e.g., from the Magnus relationship (Camuffo, 1998), as follows

$$
x=3.795 \times 10^{-3} \varphi 10^{a T /(b+T)},
$$

where $a=7.5$ and the additive temperature constant $b=237.3^{\circ} \mathrm{C}$. Only from $x$ can the actual water vapour content be assessed for a given air volume. On the other hand the relative humidity changes inversely to the temperature change if the absolute humidity $x$ is maintained at a constant level. Then for two states $\varphi_{1}, T_{1}$ and $\varphi_{2}, T_{2}$ of humid air with the same $x$ it holds

$$
\frac{\varphi_{2}}{\varphi_{1}}=\frac{10^{a T_{1} /\left(b+T_{1}\right)}}{10^{a T_{2} /\left(b+T_{2}\right)}} .
$$

Hence, if the humid air temperature has changed without changing the water vapour content $x$, its relative humidity moves in the opposite way. For example, a temperature drop under $x=$ const. necessitates the relative humidity increase given by (5). This phenomenon can be observed in simultaneous records of $\varphi$ and $T$, where most of the relative humidity fluctuations are usually due to temperature changes, while only a minor part is due to the actual change in water vapour content.

Another consequence can be derived from the Magnus formula. If a considerable change in relative humidity from $\varphi_{0}$ to $\varphi$ has occurred under $x=$ const., the corresponding temperature change is relatively small, given as follows

$$
T_{0}-T \cong \frac{b+T_{0}}{a}\left(\log \varphi-\log \varphi_{0}\right),
$$

where, unlike (3), the decimal logarithm is used. This means that, for example, a drop of relative humidity from 60 to 40 per cent, where $x$ does not change, corresponds to a temperature increase of $6{ }^{\circ} \mathrm{C}$ only. Since the sorption isotherms describing the relationship $u=\Psi(\varphi, T)$ are rather close to each other, the small temperature increments given by (6) result in the property that the lines of $x=$ const. are of a rather similar shape to the isotherms in the diagram of $u=\Psi(\varphi, T)$ and are relatively close to them. A comparison of several isotherms with the lines of $x=$ const. can be seen in Fig. 2 .

Model (3) is marked out with advantageous analytical properties, particularly with regard to its derivatives. The model parameters $A, B, C$ can be assessed to fit a set of data obtained either from experiments or from a more precise EMC model (1) or (2). For a given set of data $\varphi_{i}, T_{i}, u_{i}$, $i=1,2, \ldots, n$ the most fitting parameters $A, B, C$ can be identified by means of the least-square approach. For wood the parameter $C$ is about $0.65, B$ is from 260 to $310^{\circ} \mathrm{C}$ and the value of $A$ is about $0.28^{\circ} \mathrm{C}^{-1}$ if $u$ is expressed in $\mathrm{kg} / \mathrm{kg}$. In this way either the experimental data or the more complicated formulae (1), (2) can be fitted by the relatively simple model (3) with a good coincidence over sufficiently broad intervals, i.e. for air temperature from 5 to $40{ }^{\circ} \mathrm{C}$ and for air humidity from $10 \%$ to $90 \%$ in the controlled interior. An example of the attainable conformity of the Simpson expression of a sorption isotherm with the Henderson model is shown in Fig. 1. On the other hand, it is obvious from the nature of function (3) that it cannot fit EMC for relative humidity values close to $100 \%$, since then $\ln (1-\varphi) \rightarrow-\infty$. Obviously, due to varying sorption properties, parameters $A, B, C$ result in different values for various materials.

In order to fit the three parameters of the Henderson model to the more precise Simpson model, the iterative least square method was used. Applying this method to fit the points of the characteristics within the intervals $T \in\langle 0,25\rangle^{\circ} \mathrm{C}$ 


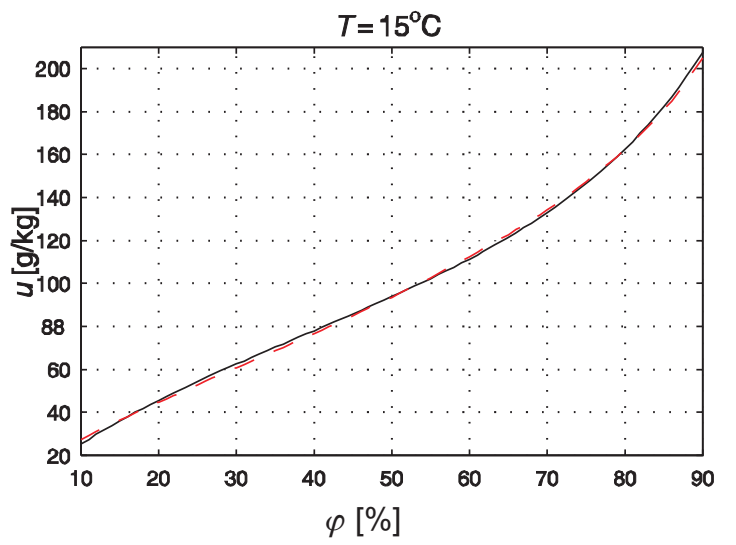

Fig. 1: Comparison of fitting the Simpson model by the Henderson model, with parameters $A=6.9610^{-5}, B=305.7$, $C=0.651$, isotherms $T=15{ }^{\circ} \mathrm{C}$ (solid - Simpson, dashed Henderson)

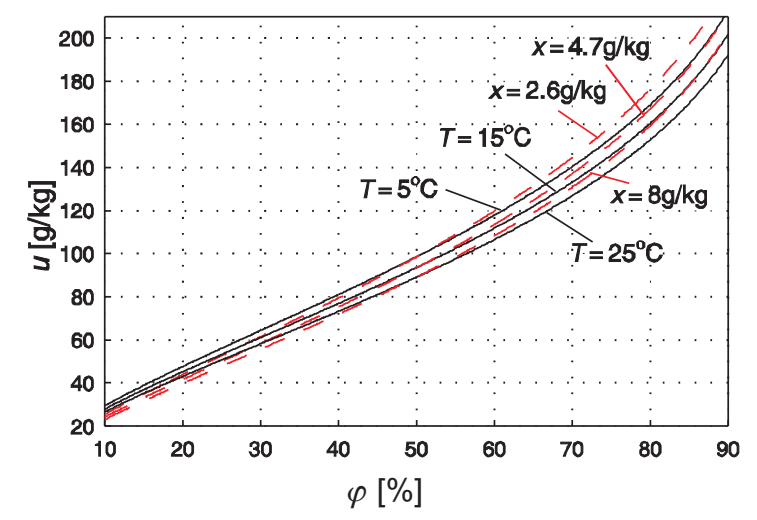

Fig. 2: Comparison of the shapes of isotherms (solid) and the lines of $x=$ const. (dashed) (using the Magnus and Henderson models)

and $\varphi \in\langle 10,90\rangle \%$, which define the region of interest from a practical point of view, the following parameters result $A=6.96210^{-5}, B=305.7^{\circ} \mathrm{C}$ and $C=0.64$. The accordance of these two models can be seen in the comparison of one of the isotherms in Fig. 1.

\section{Equal-sorption principle of air humidity control}

Let us assume the well-being of the preserved exhibits as the priority in setting up the parameters of the exhibition room microclimate. The following facts and claims are then to be taken into account in controlling the internal air parameters

- The impact of air humidity changes is more significant than the impact of usual temperature fluctuations.

- The primary harmful impact on the state of the exhibits is due to the moisture content absorbed in their porous or organic materials.

- Changes in moisture content have the crucial harmful impact, bringing about an anisotropic swelling or shrinking that results in deformations or cracks in exhibits.

- Furthermore, a higher level (>12\%) of moisture content becomes favourable for microbiologically harmful organisms to attack the surface of the exhibit.
Hence, it is not the air temperature and humidity, but the moisture content in the absorbent materials that is the decisive parameter to be kept as constant as possible in order to avoid any swelling or shrinking of the exhibits. However, this parameter can hardly be considered as a usual controlled variable, since its continual measurements are not available. First of all, the harmful impact is brought about by the anisotropic behaviour of the exhibit materials. On the other hand, it is important to realize that an exactly constant EMC can be kept only in one sort of exhibit due to the differences among the sorption isotherms for various materials. Moreover, the idea of compensating the spontaneous temperature fluctuations by humidity adjustments is feasible if, and only if, the variations of the interior temperature are smooth and slow enough, i.e. sufficiently close to a steady state. It should be emphasized that the moisture content is only slightly sensitive to temperature variations, but much more dependent on the air humidity. Let the following example be mentioned: A great temperature increase, say from $5{ }^{\circ} \mathrm{C}$ to $40{ }^{\circ} \mathrm{C}$, causes approximately the same change in moisture content in wood as results from a relative humidity drop as small as about $6 \%$, (Camuffo, 1998).

Although no direct measurement of EMC is available, the Henderson model allows us to express its value analytically from the air humidity and temperature measurement, provided that the state of the environment is all the time close to a steady state. With the help of this model, a control action can be assessed protecting exhibits made of the selected material from harmful EMC changes. Let us assume that the temperature change is slow and smooth, and let a simultaneous air humidity correction be provided in order to keep zero increment of the moisture content. In other words, although both temperature $T$ and relative humidity $\varphi$ of the air change, the moisture content in a specific material can be kept constant. If small increments of the state variables are considered, the compensating $\Delta \varphi$ correction results from the derivative of model (3), i.e. from the condition (Zítek and Němeček, 2004)

$$
\Delta u \cong \frac{\partial \Psi}{\partial T} \Delta T+\frac{\partial \Psi}{\partial \varphi} \Delta \varphi=0
$$

Using the Henderson model, the derivatives in this equation can be obtained from (3) in an analytical form. For the air temperature, the derivative is always negative

$$
\frac{\partial u}{\partial T}=\left[\frac{-\ln (1-\varphi)}{A}\right]^{C} \frac{(-C)}{(B+T)^{1+C}}<0
$$

$(0<\varphi<1)$ and, conversely, for the humidity the derivative is always positive

$\frac{\partial u}{\partial \varphi}=\left[\frac{1}{(B+T)}\right]^{C}\left[\frac{-\ln (1-\varphi)}{A}\right]^{C-1} \frac{C}{A(1-\varphi)}>0$.

The opposite signs of the two derivatives correspond to the opposite character of temperature and relative humidity increments in case of unchanged absolute humidity $x$ given as the ratio of water vapour mass to the mass of dry air. Following the requirement to keep the moisture content constant in the selected material, it is necessary to provide a compensating air humidity correction $\Delta \varphi$ corresponding to the admissible temperature increment $\Delta T$ according to the following ratio of the derivatives 


$$
\Delta \varphi=-\frac{\partial u / \partial T}{\partial u / \partial \varphi} \Delta T=-K_{C}(T, \varphi) \Delta T,
$$

where the compensation gain $K_{C}(T, \varphi)$, given by

$$
K_{C}(T, \varphi)=\frac{\ln (1-\varphi)(1-\varphi)}{B+T}
$$

is a function of both the temperature and the air humidity. However, from the three parameters of the Henderson model, $K_{C}(T, \varphi)$ is dependent only on $B$. Obviously, due to the dominant role of $B$ in the denominator value, the relative change of with respect to the temperature variations is fairly low.

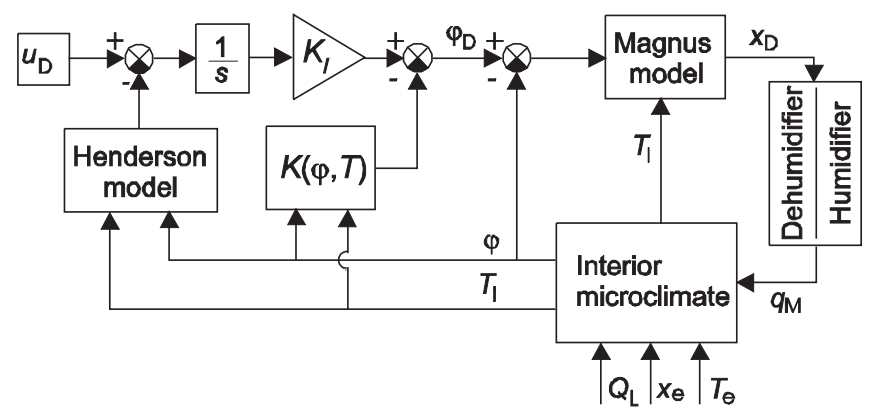

Fig. 3: Scheme of microclimate control

The increment-proportional character of relationship (11) does not guarantee that a constant desired EMC value $u=u_{D}$ can be maintained by this kind of control. In order to prevent the moisture content from drifting from $u_{D}$, the integration action has to be added into the controller and the following feed-forward-feedback control action results

$\frac{\mathrm{d} \varphi(t)}{\mathrm{d} t}=-K_{C}(\varphi(t), T(t)) \frac{\mathrm{d} T(t)}{\mathrm{d} t}+K_{I}\left[u_{D}-\Psi(T(t), \varphi(t))\right]$,

where the feed-forward gain $K_{C}(\varphi, T)$, compensating the temperature trend, is given by (11), the unavailable EMC value is computed from the $\varphi$ and $T$ measurements by means of model (3), and $K_{I}$ is an integration gain. This controller is considered as a master controller in a cascade scheme, see Fig. 3, where the slave is a humidity control with a variable desired $\varphi$ given by (12). With regard to the relative humidity to be kept in the controlled environment (within the interval $\varphi \in\langle 40,60\rangle \%)$, the weak variability of the feed-forward gain can be neglected and a constant coefficient $K_{C}(\varphi, T) \approx-0.0013{ }^{\circ} \mathrm{C}^{-1}$ can be considered. As regards the integration gain $K_{I}$, its value should be assigned low enough to prevent the control process from obtaining an oscillatory character. In any case, it is desirable to set $K_{I}$ to achieve a sufficient filtering effect in the day cycle fluctuations of the relative air humidity.

In general, the dynamics of the interior air humidity can be considered subject to the model

$V \rho \frac{\mathrm{d} x_{I}(t)}{\mathrm{d} t}=Q_{L}\left[x_{E}(t)-x_{I}(t)\right]+\beta_{x} S_{W}\left[x_{W}(t)-x_{I}(t)\right]+q_{M}(t)$

where $V$ is the interior volume, $\rho$ air density, $Q_{L}$ the in-leakage of the ambient air, $\beta_{x}$ the effective diffusion coefficient between the walls and the internal air, $S_{W}$ is the entire wall surface and $q_{M}$ the water input provided by the humidifier $\left(q_{M}>0\right)$ or dehumidifier $\left(q_{M}<0\right)$. The values of absolute humidity of the external and internal air and the walls respec- tively, are $x_{E}, x_{I}, x_{W}$, where $x_{I}$ is computed from the measured interior temperature $T(t)$ and the interior relative humidity by means of the Magnus formula (4). The in-leakage flow of the outdoor air is usually referred to as being approximately 25 to $30 \%$ of the internal volume per hour $\left(Q_{L} /(V \rho) \approx(0.25\right.$ to 0.3$\left.) \mathrm{h}^{-1}\right)$. The moisture diffusion from the walls is highly variable. According to our measurements, the moisture release from damp walls may represent as significant a water source for the interior as window leakage does. The last term $q_{M}(t)$ of (13) is the water transfer amount. This control actuating effect has to be powerful enough to counteract even the highest demands of moisture transport. The time constant of the humidity process (13) in the interior is as follows

$$
\tau=\frac{V \rho}{Q_{L}+K_{D}} .
$$

Since the on-off control is commonly used for the humidifying device (i.e. as the slave controller) this time constant is valid approximately also for the humidity-controlled interior. Unlike the master controller output, equation (13) is expressed for absolute humidity $x$, but the interior output measurement is again provided for the relative humidity. Hence the two conversions $\varphi \rightarrow x$ and $x \rightarrow \varphi$ cancel each other in the overall plant model. The only conversion left is given by the Henderson model. After linearizing this model as in (7) the following characteristic equation of the control loop is obtained

$$
\tau s^{2}+s+K_{I} \frac{\partial u}{\partial \varphi}=\tau s^{2}+s+K_{I} k_{H}=0
$$

and the non-oscillating character of the control process is obtained if the integration gain satisfies the condition $K_{I} \leq\left(4 \tau k_{H}\right)^{-1}$. In considering the usual parameters for an interior about $100 \mathrm{~m}^{2}$ in size or larger, this involves adjusting the integration gain approximately as low as $K_{I}<1 \mathrm{~h}^{-1}$.

\section{Application case study}

Within the framework of the research project several historical interiors in the Czech Republic are being monitored. One of them is St. Václav Chapel in Plasy Monastery in West Bohemia. As in the other sites the selected interior microclimate is monitored by dataloggers measuring the temperatures and relative humidities each 30 minutes.

In the Plasy application, a model of the interior EMC process has been identified from the collected data, and an equal-sorption humidity controller has been designed. Correlating the interior and exterior absolute humidity (computed by means of the Magnus formula) and using the least-square-based identification method, the time constant results at $\tau=2.44$ hours. The interval in Fig. 5 was selected for identification in order to obtain the day cycle fluctuations of the absolute humidity with the least influence of long-term transient phenomena. Due to this selection, wall moisture release, which has much slower dynamics than the variations caused by infiltration (the dynamics of which we want to model), has a minimum effect on the system dynamics. A comparison of the interior absolute humidity simulated by model (13) (the exterior absolute humidity is considered as the model input) and the real interior absolute humidity can be seen in Fig. 5 . 


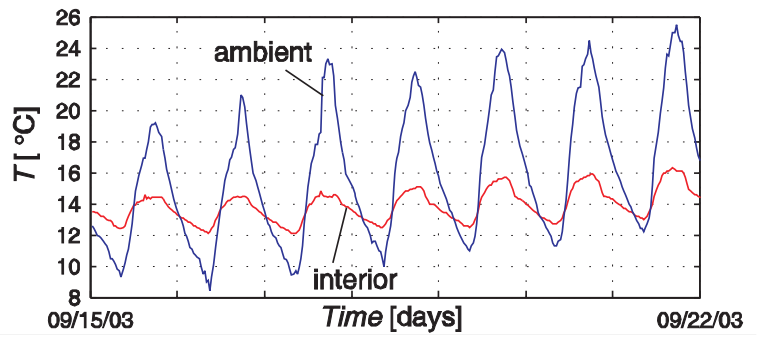

Fig. 4: Temperature in St. Václav's Chapel and ambient temperature measurements

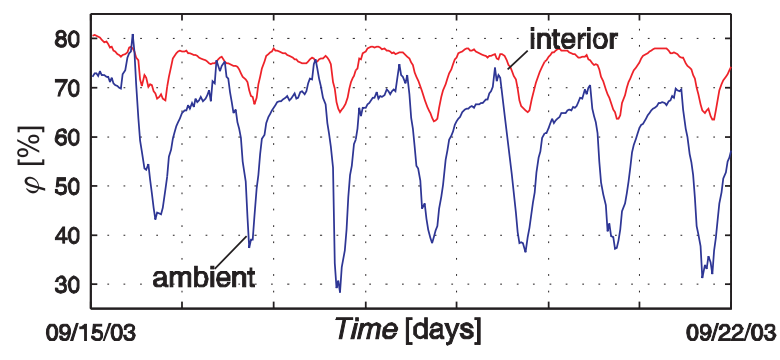

Fig. 5: Relative humidity in St. Václav's Chapel and ambient relative humidity measurements

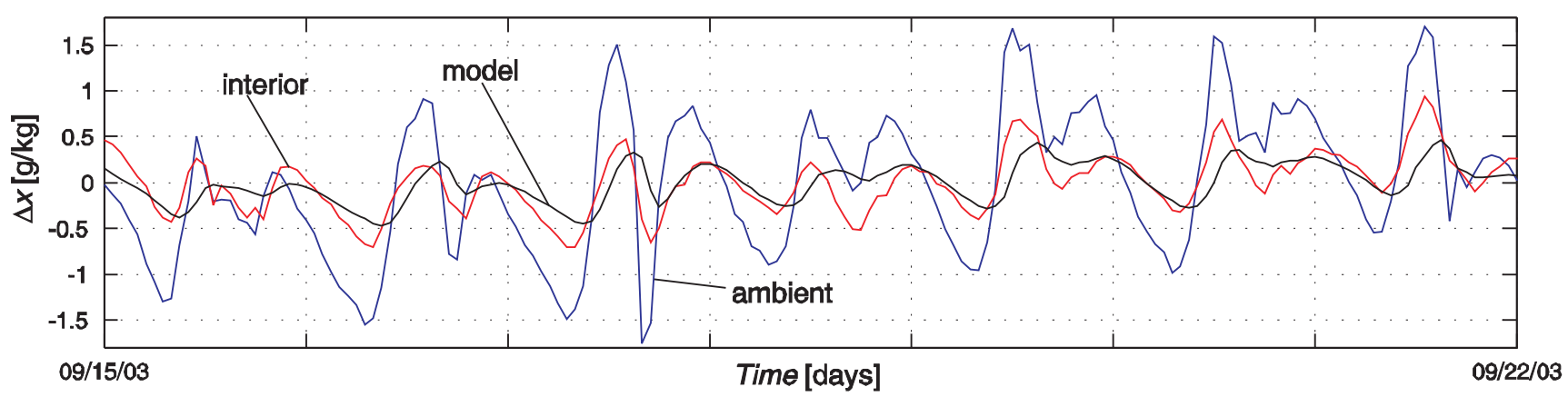

Fig. 6: Process/model comparison, model time constant $\tau=2.44$ hours and static gain $Q_{L} /\left(Q_{L}+K_{D}\right)=0.14$ (absolute humidity mean values: $\left.x_{I}=5.74 \mathrm{~g} / \mathrm{kg}, x_{E}=6.45 \mathrm{~g} / \mathrm{kg}\right)$

Even though there are considerable discrepancies between the modeled and measured data (the process itself is nonlinear and its dynamics is much more complicated than the dynamics of model (13)), the model fits the character of the dynamics sufficiently.

Using the model to transfer the measured exterior humidity into the interior humidity, and considering the interior temperature to be the temperature measured on the same time interval as the exterior humidity, see Fig. 5, the simulation of the control in keeping EMC has been performed. The results of the simulation are shown in Fig. 8. The parameters of the master controller have been chosen $K_{C}=0.0011{ }^{\circ} \mathrm{C}^{-1}, K_{I}=0.007$ hour $^{-1}$. The maximum dehu-
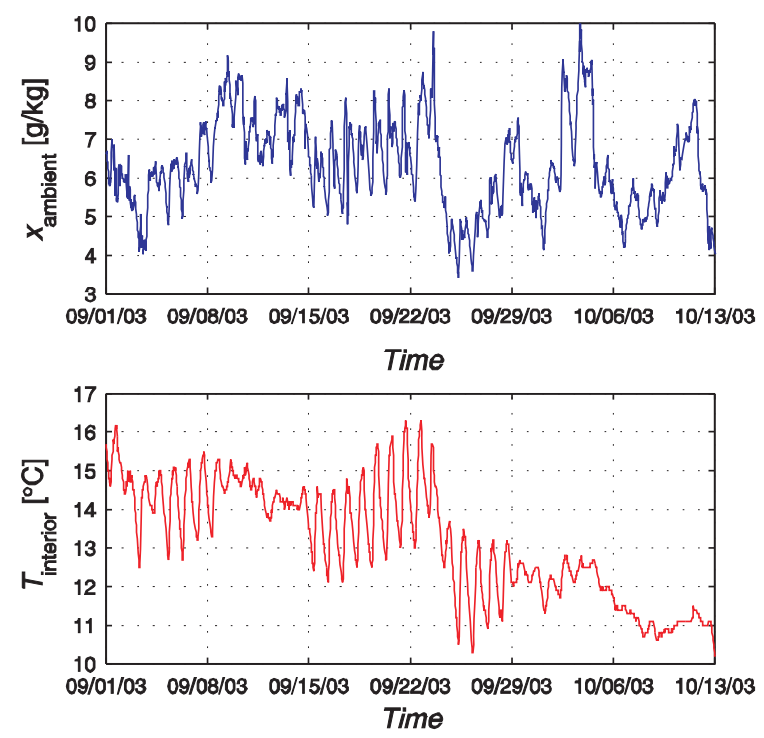

Fig. 7: The ambient relative humidity and interior temperature used as the model inputs in simulating control on EMC midifier performance needed for counteracting the worst moisture sources is approximately $500 \mathrm{~g}$ of water per hour.
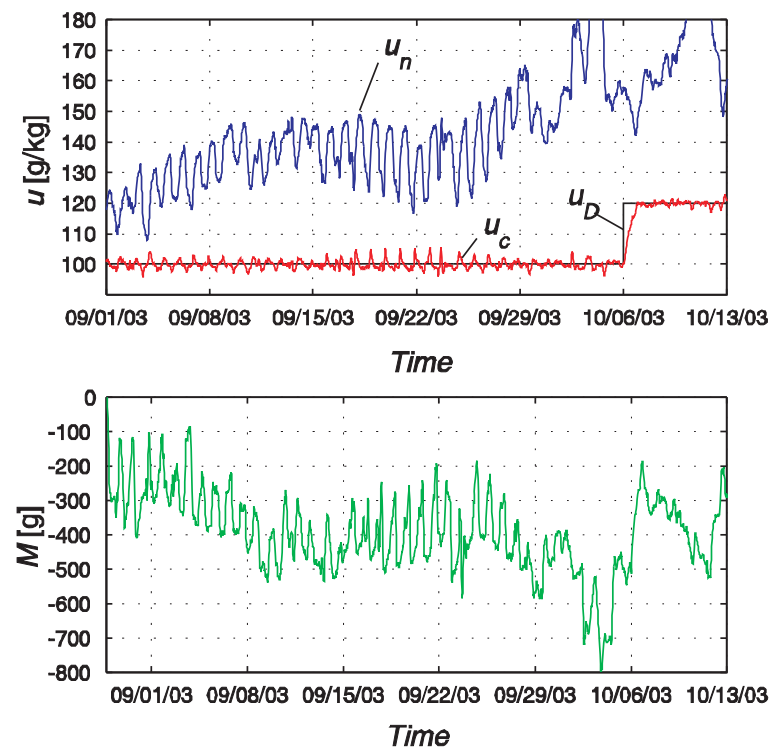

Fig. 8: Results of simulating the control on EMC in St. Václav's Chapel. Upper: $u_{n}$ - EMC if no control is used (computed from the measured data), $u_{D}$ - desired value of EMC, $u_{c}-$ EMC if control is used (resulting from simulation). Lower: the amount of water to be extracted

\section{Conclusions}

A novel microclimate control scheme has been proposed, for protecting preserved exhibits from changes in equilibrium moisture content in the materials they are made of. Although the EMC as an alternative controlled variable is unavailable for continual measurement, changes in EMC can be esti- 
mated by a non-linear model (3) from the humidity and temperature measurements. Compensating the natural interior temperature variations simultaneously by the appropriate adjustment of air humidity proves efficient in maintaining a reference EMC constant during the entire annual weather cycle. Basically, the non-linear model-based microclimate control is marked out with a feed-forward character since the changes in the thermodynamic air state variables are much faster than the sorption phenomena, and therefore they inhibit the possible sorption process. The proposed moisture content control scheme has already been implemented and has been in successful operation in The Holy Cross Chapel at Karlštejn Castle in the Czech Republic (Zítek and Němeček, 2004) since 1999. Incited by the alarming deterioration of the collection of 29 precious medieval paintings by Master Theodoricus, the implementation was based on a thorough investigation of the interior microclimate. In heavy inertial structures, like those in this implementation, the temperature course can be allowed to run almost its natural yearly cycle, without considerable heating influence, since the temperature does not drop below six or seven ${ }^{\circ} \mathrm{C}$. However, in mansions and similar buildings which do not have such thick walls, the winter course of the heating-unaffected interior temperature would drop to zero, or even below freezing point during the winter season, and it is essential to maintain the temperature safely above zero. Hence the temperature cycle has to be corrected by a low-level heating during the winter season in buildings where such low temperatures occur. On the other hand, moisture control is able to safely protect the interior from condensation effects if the interior temperature does not drop below $5{ }^{\circ} \mathrm{C}$.

Although the proposed control scheme is based on the EMC model for a single material selected as decisive for preservation purposes, the differences between the sorption isotherms of various materials affect the control action only weakly, also with regard to the low level of humidity corrections needed. Humidity compensation therefore works well not only for the selected material but also for other materials. Although the sorption differences between materials are expressed in three parameters $A, B, C$, only $B$ (in ${ }^{\circ} \mathrm{C}$ ) influences the main part of the control action (13). Only this property of the Henderson model turned out to be significant in helping to show that the control actions (13) computed for various materials would not be very distinct. The value of $B$ is relatively high (more than $200^{\circ} \mathrm{C}$ ) and therefore the compensation control parameter $K_{C}(T, \varphi)$ is rather low and makes the humidity correction relatively small. In this way the sorption differences between various materials have an inconsiderable effect on the resulting control action (13). On the other hand, the following two factors are to be emphasized:

- The equilibrium assumption. It is of primary importance to avoid any rapid air temperature and humidity changes in the interior.

- Providing sufficient internal air circulation. The control of the internal air humidity will have little effect without ensuring sufficient air circulation, particularly near the surface of the walls.

It should be emphasized that the most important advantage of equal-sorption control is not economy in power consumption but, above all, the careful character of its opera- tion from the preventive conservation point of view. Due to the lower temperature and controlled humidity during the winter season the exhibits do not suffer such exposure to high gradients of both temperature and humidity as they do in the case of usual air-conditioning, or in historical buildings where no technical care of the internal environment is provided.

\section{Acknowledgement}

The presented research has been supported by the Grant Agency of the Czech Republic under Project No. 101-03-1365 and by the Ministry of Education of the Czech Republic under Project LN00B096.

\section{References}

[1] Camuffo, D.: Microclimate for Cultural Heritage. Elsevier Science Ltd., Amsterdam, London, 1998

[2] Camuffo, D., Bernardi, A., Sturaro, G., Valentino, A.: "The Microclimate inside the Pollaiolo and Botticelli Rooms in the Uffizi Gallery, Florence." Journal for Cultural Heritage, Vol. 3 (2002), p. 155-161.

[3] Cassar, M.: Environmental Management. Guidelines for Museums and Galleries. Routledge, London 1995

[4] Cassar, M.: "A Pragmatic Approach to Environmental Improvements in the Courtauld Institute Galleries in Somerset House." ICOM Committee for Conservation, Vol. 2 (1993), p. 595-600.

[5] Kotterer, M.: "Research Report of the Project EU 1383 PREVENT”, Museum Ostdeutsche Galerie Regensburg, 2002.

[6] Kowalski, S. J.: Thermomechanics of Drying Processes. Springer, Berlin, 2003.

[7] Massari, G., Massari I.: Damp Buildings. Old and New. ICCROM, Rome, 1993.

[8] Ball, R. D., Simpson, I. G. and Pang, S.: "Measurement, Modelling and Prediction of E.M.C. in Pinus Radiata Heartwood and Sapwood." Holz als Roh- und Werkstoff 59 (2001), Springer-Verlag 2001, p. 457-462.

[9] Zítek, P., Němeček, M.: "Stabilizing Microclimate of Historical Interior by Moisture Sorption Control.” IEEE Conference on Math. Modeling, Automation and Robotics 2004, Szczecin, Poland (to appear).

Prof. Ing. Pavel Zítek, DrSc. e-mail: zitek@fsid.cvut.cz

Ing. Tomáš Vyhlídal, Ph.D. e-mail:vyhlidal@fsid.cvut.cz

Centre for Applied Cybernetics

Institute of Instrumentation and Control Engineering

Czech Technical University in Prague

Faculty of Mechanical Engineering

Technická 4

16607 Praha 6, Czech Republic 